\title{
ON ISOPTICS AND ISOCHORDAL-VIEWED CURVES
}

\author{
DAVID ROCHERA
}

\begin{abstract}
In this paper, some results involving isoptic curves and constant $\phi$-width curves are given for any closed curve. The non-convex case, as well as non-simple shapes with or without cusps are considered. Relating the construction of isoptics to the construction given in Holditch's theorem, a kind of curves is defined: the isochordal-viewed curves. The explicit expression of these curves is given together with some examples. Integral formulae on the area of their isoptics are obtained and a Barbier-type theorem is derived. Finally, a characterization for isochordal-viewed hedgehogs and curves of constant $\phi$-width is given in terms of an angle function.
\end{abstract}

\section{INTRODUCTION}

1.1. Definition of isoptic curves. Consider first a regular convex planar curve $\alpha:[0,2 \pi] \rightarrow \mathbb{R}^{2}$ defined by a support function $h \in \mathcal{C}^{2}$. This is,

$$
\alpha(t)=h(t)(\cos t, \sin t)+h^{\prime}(t)(-\sin t, \cos t) .
$$

Denote by $\mathbf{t}$ and $\mathbf{n}=J \mathbf{t}$ the tangent and normal vectors, respectively, of $\alpha$ at $\alpha(t)$.

Given $\phi \in] 0, \pi[$, the $\phi$-isoptic of $\alpha$ is the locus of points from which the curve $\alpha$ is seen under a constant angle $\pi-\phi$ (see for instance [4] and [5]). For each $t \in[0,2 \pi]$, the supporting lines that contain the curve $\alpha$ under this constant angle $\pi-\phi$ are those that touch the curve at the points $\alpha(t)$ and $\alpha(t+\phi)$ (see Figure 11). Denote by $\lambda_{1}(t, \phi)$ the distance from $\alpha(t)$ to the $\phi$-isoptic and by $\lambda_{2}(t, \phi)$ the same from $\alpha(t+\phi)$.

Thus, the $\phi$-isoptic of $\alpha$ can be written in two different ways as a curve $\alpha_{\phi}:[0,2 \pi] \rightarrow \mathbb{R}^{2}$ defined by:

$$
\begin{aligned}
\alpha_{\phi}(t) & =\alpha(t)+\lambda_{1}(t, \phi) \mathbf{t}(t) \\
& =\alpha(t+\phi)-\lambda_{2}(t, \phi) \mathbf{t}(t+\phi),
\end{aligned}
$$

Key words and phrases. Isoptic curves, Isochordal-viewed curves, Holditch's theorem, Curves of constant $\phi$-width, Hedgehogs.

The author has been partially funded by the BCAM Severo Ochoa accreditation of excellence, Spain (SEV-2017-0718).

This is a preprint of an article published in Aequationes Mathematicae. The final authenticated version is available online at: https://doi.org/10.1007/s00010-021-00835-5 


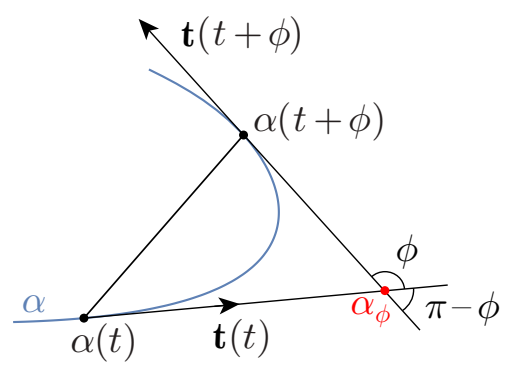

Figure 1. Definition of the $\phi$-isoptic $\alpha_{\phi}$ of $\alpha$.

Isoptic curves have been studied thoroughly for smooth convex curves in the last decades (see for instance the cited papers [4] and [5], among many others by the same authors). If $\alpha$ is a strictly convex curve, then for any point in the outside region determined by $\alpha$ there exists a unique pair of tangents to $\alpha$. This is essential to define properly isoptic curves. The angle $\phi$ in the definition of its $\phi$-isoptic can be computed easily as the angle between these two tangents.

Nevertheless, just a few references on isoptics for non-convex curves are found in the literature. In the paper [7] a symbolic study on the isoptics of an astroid was performed. Astroid curves are examples of non-convex curves with cusps. In this example it was shown that there exist points where three tangents to the astroid are found. If a pre-definite angle is considered, namely $\phi$, the third tangent is irrelevant as it is pointed out in the same paper. Doing a careful study of the astroid example, we can see that the angle between the tangents does not remain exactly constant. See Figure 2; at the beginning, the angle between the tangents is indeed $\phi$, but when one of the tangents passes through one of the cusps, then the angle between the tangents turns into $\pi-\phi$. This would go against the definition of an isoptic which demands the curve to be seen under a constant angle. In order to avoid this issue for non-convex curves, we take the definition of an isoptic given in recent papers such as [7] or [8], which can be stated in the following manner.

Definition 1 (Isoptic curve). Given a planar curve $\alpha$ and an angle $\phi \in] 0, \pi[$, define the $\phi$-isoptic of $\alpha$ as the curve defined by the locus of points through which passes a pair of tangents to $\alpha$ making an angle of $\phi$.

Note that this definition allows the angle between the tangents to be $\phi$ or $\pi-\phi$.

1.2. Sliding a constant length chord seen under a constant angle. In the non-convex case, the definition of the $\phi$-isoptic with the distance functions $\lambda_{1}$ or $\lambda_{2}$ as in (1) must be written carefully, since these two distance functions may include sign changes. The consideration of these sign changes 

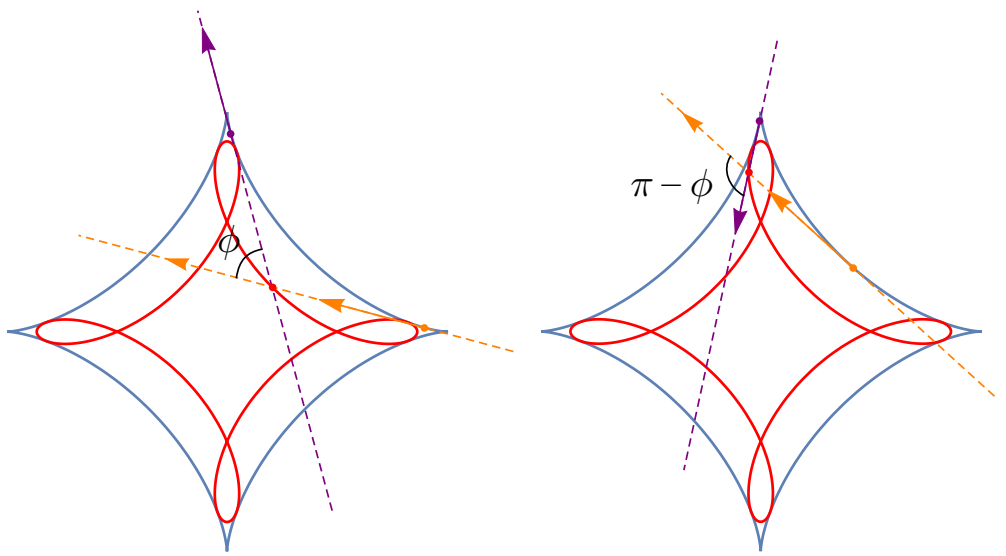

Figure 2. The astroid curve $\left(\cos ^{3} t, \sin ^{3} t\right)$ and its $\phi$-isoptic, for $\phi=\pi / 3$. The angle between the tangent lines is piecewise-constant equal to $\pi / 3$ or $2 \pi / 3$.

is essential for non-convex curves, because they may happen due to nonsimple shapes or cusps, for example. The sign is dependent on the oriented angle from one tangent to the other, which may be either $\phi$ or $\pi-\phi$ up to a multiple of $\pi$. This will be discussed later in Proposition 1 .

In [11, the authors worked with a special kind of convex bodies. Let $K$ be a strictly convex body in $\mathbb{R}^{2}$ with differentiable boundary and let $\left.\phi \in\right] 0, \pi[$. A straight segment connecting two points of this boundary is called a $\phi$ chord if the tangent lines to $K$ at these two points intersect at an angle $\phi$. In the cited paper, the authors considered convex bodies with $\phi$-chords of constant length. Some interesting partial results were given (under some conditions) towards the Euclidean disc being the only example of this kind of convex bodies (the general result is still open).

In this paper, we will study precisely the kind of curves given by the boundary of such sets, but not restricted to the convex case. Recall that the sliding of a constant length chord around a given curve corresponds to the famous setting studied by Hamnet Holditch in 1858, [10, and by many other authors in papers such as [1, [6] or, more recently, in the works [3, [14] and [17]. In fact, in [3] the reader can find some results on sliding chords in the construction of isoptics and a new result on areas of ring domains in a modified Holditch construction.

To sum up, we will give a name to the kind of curves to be considered in this work.

Definition 2 (Isochordal-viewed curve). Given $\phi \in] 0, \pi[$ and $\ell>0$, a planar curve $\alpha$ is said to be $(\phi, \ell)$-isochordal viewed if the $\phi$-isoptic of $\alpha, \alpha_{\phi}$, is such that the chord joining the contact points with $\alpha$ of its supporting lines meeting at $\alpha_{\phi}$ has constant length $\ell$. 
The obvious example of a curve where a constant length chord can travel around and which can always be seen under a constant angle is the circle (and it might be the only convex example, but this is just a conjecture for now). Nevertheless, in the non-convex case other examples different from the circle can be given and the definition does not become trivial. In fact, in Examples 1, 2 we will study two hedgehogs which are isochordal-viewed. Moreover, a 1-parameter family of examples will be given in Example 3 .

The paper is structured as follows. First, in Section 2, the general setting for isochordal-viewed curves is addressed and the explicit expression for the $\phi$-isoptic of a given closed curve is obtained (Proposition 1). The proof of this expression leads to a generalization of the so-called sine-theorem for isoptics (Remark 1). In Section 3, the definition of curves of constant $\phi$ width is recalled and some examples of $(\phi, \ell)$-isochordal-viewed curves and curves of constant $\phi$-width are constructed. After that, in Section 4 we derive some formulae on the area of the isoptics of isochordal-viewed curves (Propositions 2 and 3), together with a Barbier-type theorem for isochordalviewed curves (Theorem 1). Finally, in Section 5 a characterization of when a isochordal-viewed hedgehog is a curve of constant $\phi$-width is given (Theorem 2).

\section{General Setting for isochordal-VieWed Curves}

Henceforth, let $\alpha: I \rightarrow \mathbb{R}^{2}$ be a piecewise-regular closed planar curve, where $I$ is some real interval (and assume that the curve can be extended to the whole $\mathbb{R}$ by periodicity). Recall that the Holditch function $f: I \rightarrow f(I)$, see [14], is defined as the homeomorphism that given the rear endpoint $\alpha(t)$ of the sliding chord produces the front one, $\alpha(f(t))$. Note that the definition of $f$ depends on the chosen parameterization of $\alpha$ and it assumes that no retrograde movements are done by the moving chord.

Henceforth, given a curve $\alpha: I \rightarrow \mathbb{R}^{2}$ and an angle $\left.\phi \in\right] 0, \pi[$, we will define $f: I \rightarrow f(I)$ such that $\alpha(t)$ and $\alpha(f(t))$ are the contact points of $\alpha$ where two tangent lines to $\alpha$ meet at an angle $\phi$. This function will be called the Holditch function for the parameterization $\alpha$ and the angle $\phi$. For $(\phi, \ell)$-isochordal-viewed hedgehogs parameterized by a support function, this Holditch function is just a translation by the angle $\phi: f(t)=t+\phi$.

Define the distance function $d: I \rightarrow[0,+\infty[$ as

$$
d(t):=\|\alpha(f(t))-\alpha(t)\| .
$$

In the situation we are addressing to, where the constant length is $\ell$, we have the constraint:

$$
d(t):=\|\alpha(f(t))-\alpha(t)\|=\ell, \quad \text { for all } t \in I .
$$

We will call this constraint as the isochordal condition. 
Define $\nu(t)$ as the oriented angle function from $\mathbf{t}(t)$ to $\alpha(f(t))-\alpha(t)$ and $\mu(t)$ as the oriented angle function from $\alpha(f(t))-\alpha(t)$ to $\mathbf{t}(f(t))$. Assume that the starting angle at $\inf I$ is oriented in $]-\pi, \pi]$. Note that, again, the angles $\nu$ and $\mu$ depend on the chosen parameterization $\alpha$. From now on, $\nu$ and $\mu$ will denote these two oriented angle functions according to the chosen parameterization (see Figure 3 for a visualization of these angle functions).

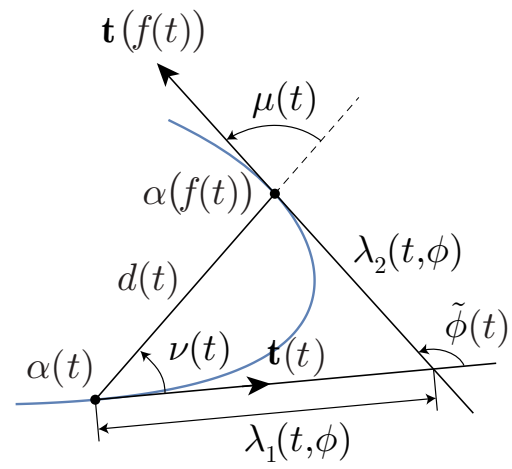

Figure 3. Definition of the Holditch function $f$, the angles $\nu, \mu$ and $\phi$, and the distance functions $d, \lambda_{1}$ and $\lambda_{2}$.

By definition of $\nu$, we have:

$$
\alpha(f(t))-\alpha(t)=d(t)(\cos \nu(t) \mathbf{t}(t)+\sin \nu(t) \mathbf{n}(t)) .
$$

Analogously, by definition of $\mu$ we can deduce:

$$
\alpha(f(t))-\alpha(t)=d(t)(\cos \mu(t) \mathbf{t}(f(t))-\sin \mu(t) \mathbf{n}(f(t))) .
$$

Define

$$
\tilde{\phi}(t):=\nu(t)+\mu(t) .
$$

We have that $\tilde{\phi}$ is a piecewise-constant function (either $\phi$ or $\pi-\phi$ up to a multiple of $\pi$ ), which gives an oriented angle from $\mathbf{t}(t)$ to $\mathbf{t}(f(t))$. Thus,

$$
\begin{aligned}
\mathbf{t}(f(t)) & =\cos \tilde{\phi}(t) \mathbf{t}(t)+\sin \tilde{\phi}(t) \mathbf{n}(t), \\
\mathbf{n}(f(t)) & =-\sin \tilde{\phi}(t) \mathbf{t}(t)+\cos \tilde{\phi}(t) \mathbf{n}(t),
\end{aligned}
$$

The relation between $\tilde{\phi}$ and $\phi$ is the following:

$$
\phi=|\tilde{\phi}(t) \quad \bmod \pi|, \quad \text { for any } t \in I .
$$

In the convex case, $\tilde{\phi}(t)=\phi$ for all $t \in I$.

The functions $\lambda_{1}$ and $\lambda_{2}$ can be computed geometrically by the cosine rule (see Figure 3). Nevertheless, the computations must be carried out carefully because of possible angle changes and signs. Instead, we will derive the expression of the $\phi$-isoptic of $\alpha$ by finding the intersection point between 
the tangent lines at $\alpha(t)$ and $\alpha(f(t))$. We will do it in the general scenario of any curve, not necessarily verifying the isochordal condition.

Proposition 1. Let $\ell>0, \phi \in] 0, \pi\left[\right.$ and let $\alpha: I \rightarrow \mathbb{R}^{2}$ be a regular curve. The $\phi$-isoptic of $\alpha$ can be written as

$$
\begin{aligned}
\alpha_{\phi}(t) & =\alpha(t)+\lambda_{1}(t, \phi) \mathbf{t}(t) \\
& =\alpha(f(t))-\lambda_{2}(t, \phi) \mathbf{t}(f(t)),
\end{aligned}
$$

where $f: I \rightarrow f(I)$ is the Holditch function for the parameterization $\alpha$ and the angle $\phi$,

$$
\lambda_{1}(t, \phi)=\frac{d(t) \sin \mu(t)}{\sin \tilde{\phi}(t)} \quad \text { and } \quad \lambda_{2}(t, \phi)=\frac{d(t) \sin \nu(t)}{\sin \tilde{\phi}(t)},
$$

with $d(t)$ being the distance from $\alpha(t)$ to $\alpha(f(t))$ and with $\tilde{\phi}(t)=\nu(t)+\mu(t)$.

Proof. By definition,

$$
d(t):=\|\alpha(f(t))-\alpha(t)\| .
$$

Let $\alpha(t)=(x(t), y(t))$. The tangent vector of $\alpha$ at $\alpha(t)$ can be written as

$$
\mathbf{t}(t)=\left(\frac{x^{\prime}(t)}{\sqrt{x^{\prime 2}(t)+y^{\prime 2}(t)}}, \frac{y^{\prime}(t)}{\sqrt{x^{\prime 2}(t)+y^{\prime 2}(t)}}\right) .
$$

The condition to find the intersection point between the tangent lines to $\alpha$ at $\alpha(t)$ and $\alpha(f(t))$, namely

$$
\alpha(t)+\lambda_{1}(t, \phi) \mathbf{t}(t)=\alpha(f(t))-\lambda_{2}(t, \phi) \mathbf{t}(f(t)),
$$

produces a system of two equations with unknowns $\lambda_{1}(t, \phi)$ and $\lambda_{2}(t, \phi)$. By solving it, we find the expression of $\lambda_{1}(t, \phi)$ :

$$
\frac{\sqrt{x^{\prime 2}(t)+y^{\prime 2}(t)}\left(-(y(f(t))-y(t)) x^{\prime}(f(t))+(x(f(t))-x(t)) y^{\prime}(f(t))\right)}{x^{\prime}(t) y^{\prime}(f(t))-y^{\prime}(t) x^{\prime}(f(t))},
$$

and the same of $\lambda_{2}(t, \phi)$ :

$$
\frac{\sqrt{x^{\prime 2}(f(t))+y^{\prime 2}(f(t))}\left(-(y(f(t))-y(t)) x^{\prime}(t)+(x(f(t))-x(t)) y^{\prime}(t)\right)}{y^{\prime}(t) x^{\prime}(f(t))-x^{\prime}(t) y^{\prime}(f(t))} \text {. }
$$

These two expressions can be simplified. The first one can be written as

$$
\begin{aligned}
\lambda_{1}(t, \phi) & =\frac{-\left\|\alpha^{\prime}(t)\right\|\left\langle\alpha(f(t))-\alpha(t), J \alpha^{\prime}(f(t))\right\rangle}{-\left\langle\alpha^{\prime}(t), J \alpha^{\prime}(f(t))\right\rangle} \\
& =\frac{\langle\alpha(f(t))-\alpha(t), \mathbf{n}(f(t))\rangle}{\langle\mathbf{t}(t), \mathbf{n}(f(t))\rangle}
\end{aligned}
$$


From Equations (4) and (5), we get

$$
\langle\alpha(f(t))-\alpha(t), \mathbf{n}(f(t))\rangle=-d(t) \sin \mu(t)
$$

and

$$
\langle\mathbf{t}(t), \mathbf{n}(f(t))\rangle=-\sin \tilde{\phi}(t) .
$$

Therefore, we conclude

$$
\lambda_{1}(t, \phi)=\frac{d(t) \sin \mu(t)}{\sin \tilde{\phi}(t)} .
$$

Similarly, with Equations (3) and (5), we get

$$
\begin{aligned}
\lambda_{2}(t, \phi) & =\frac{-\left\|\alpha^{\prime}(f(t))\right\|\left\langle\alpha(f(t))-\alpha(t), J \alpha^{\prime}(t)\right\rangle}{-\left\langle\alpha^{\prime}(f(t)), J \alpha^{\prime}(t)\right\rangle} \\
& =\frac{\langle\alpha(f(t))-\alpha(t), \mathbf{n}(t)\rangle}{\langle\mathbf{t}(f(t)), \mathbf{n}(t)\rangle}=\frac{d(t) \sin \nu(t)}{\sin \tilde{\phi}(t)} .
\end{aligned}
$$

That completes the proof.

Remark 1 (Sine-theorem for isoptics). Notice that the computation of the distance functions $\lambda_{1}$ and $\lambda_{2}$ in Proposition 1 constitutes a generalization to any closed curve of the sine-theorem for convex isoptics given in [4]: it holds

$$
\frac{d(t)}{\sin \tilde{\phi}(t)}=\frac{\lambda_{1}(t, \phi)}{\sin \mu(t)}=\frac{\lambda_{2}(t, \phi)}{\sin \nu(t)}
$$

Moreover, notice that the $\phi$-isoptic of a piecewise- $\mathcal{C}^{2}$ curve is piecewise- $\mathcal{C}^{2}$.

Remark 2. For $(\phi, \ell)$-isochordal-viewed curves, the conclusion of Proposition 1 is the same but for a constant length function $d(t)=\ell$, i.e.

$$
\lambda_{1}(t, \phi)=\frac{\ell \sin \mu(t)}{\sin \tilde{\phi}(t)} \quad \text { and } \quad \lambda_{2}(t, \phi)=\frac{\ell \sin \nu(t)}{\sin \tilde{\phi}(t)},
$$

Remark 3. Since

$$
\tilde{\phi}(t)=\left\{\begin{array}{l}
\phi+k \pi, \quad k \in \mathbb{Z} \\
\text { or } \\
\pi-\phi+k \pi, \quad k \in \mathbb{Z}
\end{array}\right.
$$

we can write

$$
\sin \tilde{\phi}(t)=\varepsilon(t) \sin \phi,
$$

where $\varepsilon$ is a sign function. The use of this alternative expression for the sine of $\tilde{\phi}(t)$ in terms of the sign function $\varepsilon(t)$ will be useful later in Section 4 . 


\section{Curves of CONStant $\phi$-Width And some EXamples}

An oval is defined as a closed convex $\mathcal{C}^{2}$ curve with non-vanishing curvature. In Equation (5.7) of [4] the expression of the curvature of the $\phi$-isoptic of an oval $\alpha$ was given for a parameterization by a support function. Following the same notation as the authors, this curvature can be written as

$$
\kappa_{\alpha, \phi}(t)=\sin \phi \kappa_{\phi}(t)
$$

where

$$
\kappa_{\phi}(t)=\frac{2|q(t)|^{2}-\left[q(t), q^{\prime}(t)\right]}{|q(t)|^{3}},
$$

with $q(t)=\alpha(t)-\alpha(t+\phi)$ and $[a+b \mathbf{i}, c+d \mathbf{i}]=a d-b c$.

In this setting, Mozgawa gave in [16] a generalized notion of constant width based on the definition of a curve of constant angle (the reader can see [9] and [13]). Given an oval $\alpha$ and a $\phi$-isoptic of $\alpha$, with $0<\phi<\pi$, the function $\kappa_{\phi}$ above is called the sine-curvature of the $\phi$-isoptic of $\alpha$. An oval $\alpha$ is called of constant $\phi$-width if $\kappa_{\phi}$ is constant.

Note that this definition implies the following: an oval $\alpha$ is of constant $\phi$ width if and only if its $\phi$-isoptic is a circle. This holds true even for the case $\phi=\pi$, which corresponds to the classical constant width curves (see [16] for more details). The characterization above motivates the next definition for any closed curve, convex or not.

Definition 3 (Curve of constant $\phi$-width). A closed curve is called a curve of constant $\phi$-width if its $\phi$-isoptic is a circle.

Green showed in [9] that there are examples, different from the circle, of curves of constant angle. But what happens if instead of imposing $\kappa_{\phi}$ being constant, we set the isochordal condition (2)? In the next examples we show that there are examples different from the circle too.

Example 1. Consider the hedgehog $\alpha:[0, \pi] \rightarrow \mathbb{R}^{2}$ defined by the support function $h(t)=\cos (3 t)$ and let $\phi=\pi / 2$. Since $\pi-\phi=\phi$, the angle $\phi$ can be recovered easily as the angle between the tangents $\mathbf{t}(t)$ and $\mathbf{t}(t+\phi)$ for all $t \in I$.

We have that the isochordal condition is satisfied: the chord joining $\alpha(t)$ and $\alpha(t+\phi)$ for each $t \in[0, \pi]$ has constant length $\ell=4$, i.e.

$$
\ell=\|\alpha(t+\phi)-\alpha(t)\|=4 .
$$

Hence, $\alpha$ is a $(\phi, \ell)$-isochordal-viewed curve. Nevertheless, $\alpha$ is different from a circle (see Figure 4). 
Notice that in this case the curve $\alpha$ is negatively (clockwise) oriented. The oriented angle functions $\nu$ and $\mu$ can be computed explicitly:

$$
\begin{aligned}
& \mu(t)=\arccos \left(-\frac{\cot (t)\left(\cot ^{2}(t)-3\right) \sqrt{\sin ^{2}(3 t)}}{3 \cot ^{2}(t)-1}\right)-\pi, \\
& \nu(t)=\arccos \left(-\frac{\tan (t)\left(\tan ^{2}(t)-3\right) \sqrt{\cos ^{2}(3 t)}}{3 \tan ^{2}(t)-1}\right)-\pi .
\end{aligned}
$$

The angle function $\mu$ is increasing while $\nu$ is decreasing and they are both piecewise-linear functions. We have that

$$
\tilde{\phi}(t)= \begin{cases}-\frac{3 \pi}{2}, & \text { if } \quad t \leq \frac{\pi}{6} \quad \text { or } \quad \frac{\pi}{3}<t \leq \frac{\pi}{2} \quad \text { or } \quad \frac{2 \pi}{3}<t \leq \frac{5 \pi}{6}, \\ -\frac{\pi}{2}, & \text { if } \quad \frac{\pi}{6}<t \leq \frac{\pi}{3} \quad \text { or } \quad \frac{\pi}{2}<t \leq \frac{2 \pi}{3} \quad \text { or } \quad t>\frac{5 \pi}{6} .\end{cases}
$$

In this case, $\alpha$ exhibits three cusps in $[0, \pi]$, namely, at $t=\pi / 6, t=\pi / 2$ and $t=5 \pi / 6$. This makes a change of orientation in the tangent vector of $\alpha$ when crossing each cusp. In addition, at $t=\pi / 3, t=2 \pi / 3$ and $t=\pi$, the other tangent goes trough each cusp and there, the first tangent crosses on the other side of the tangent line defined by the second one (see Figure 4). That produces a change of sign in the definition of the $\phi$-isoptic of $\alpha$ which is contained in the definition of $\tilde{\phi}$. By Proposition 1, the $\phi$-isoptic of $\alpha$ can be written as

$$
\alpha_{\phi}(t)=\alpha(t)+\frac{\ell \sin \mu(t)}{\sin \tilde{\phi}(t)} \mathbf{t}(t) .
$$

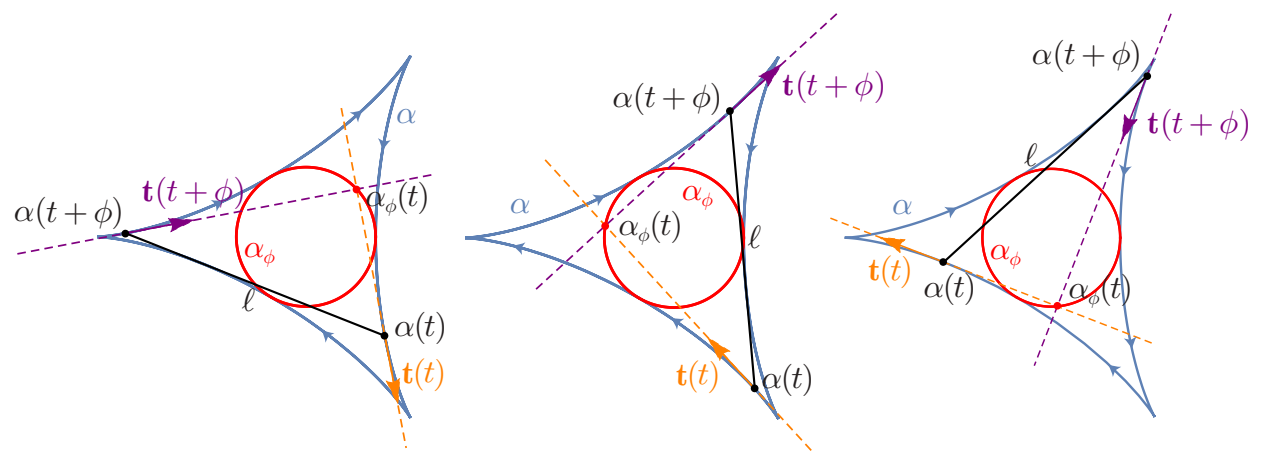

FiguRE 4 . The curve $\alpha$ is $(\pi / 2,4)$-isochordal viewed and it is different from a circle. The pointing of the tangent vectors of $\alpha$ change when crossing each cusp and it produces a change of sign in the definition of the $\pi / 2$-isoptic $\alpha_{\pi / 2}$ of $\alpha$, which turns out to be a circle of radius 1 .

In this case, the $\phi$-isoptic of $\alpha$ turns out to be a circle (counterclockwise oriented and double traced), so that $\alpha$ is also a curve of constant $\phi$-width. 
Moreover, its isoptic coincides with the Holditch curve generated by the midpoint of the moving chord (see Figure 5). The midpoint Holditch curve can be written as

$$
H_{\alpha}(t)=\frac{1}{2}(\alpha(t)+\alpha(t+\phi)) .
$$

In this example, the relation between the parametric curves $H_{\alpha}$ and $\alpha_{\phi}$ is the following:

$$
H_{\alpha}\left(t-(-2 \arctan (1-\sqrt{2}))+\frac{\pi}{2}\right)=\alpha_{\phi}(t) .
$$

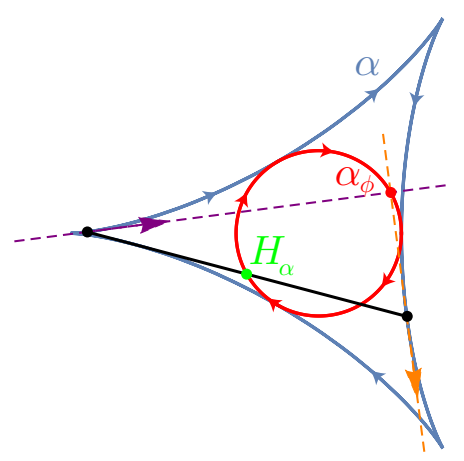

Figure 5. The $\phi$-isoptic of $\alpha, \alpha_{\phi}$, coincides with the Holditch curve of $\alpha, H_{\alpha}$. The relation between both parameterizations is just a translation.

Example 2. Consider the hedgehog $\alpha:[0, \pi] \rightarrow \mathbb{R}^{2}$ defined by the support function $h(t)=\cos (5 t)$ and let $\phi=\pi / 3$. The curve $\alpha$ is negatively (clockwise) oriented.

The isochordal condition is satisfied:

$$
\ell=\|\alpha(t+\phi)-\alpha(t)\|=3 \sqrt{3}
$$

Hence, $\alpha$ is a $(\phi, \ell)$-isochordal-viewed, which is a curve different from a circle too (see Figure 6).

The $\phi$-isoptic of $\alpha$ can be computed, by Proposition 1, as

$$
\alpha_{\phi}(t)=\alpha(t)+\frac{\ell \sin \mu(t)}{\sin \tilde{\phi}(t)} \mathbf{t}(t)
$$

The oriented angle functions $\nu$ and $\mu$ are again piecewise-linear and can be computed explicitly. Thus, computing the angle function $\tilde{\phi}(t)=\nu(t)+\mu(t)$, we can see that it is equal to either $\frac{\pi}{3}$ or $\frac{2 \pi}{3}$ up to a multiple of $\pi$. The curve $\alpha_{\phi}$ turns out to be a circle (counterclockwise and triple traced), so that $\alpha$ is a curve of constant $\pi / 3$-width.

In this case, the $\phi$-isoptic of $\alpha$ does not coincide with any Holditch curve of $\alpha$ (see an example in Figure 7fleft). If we consider the same example 

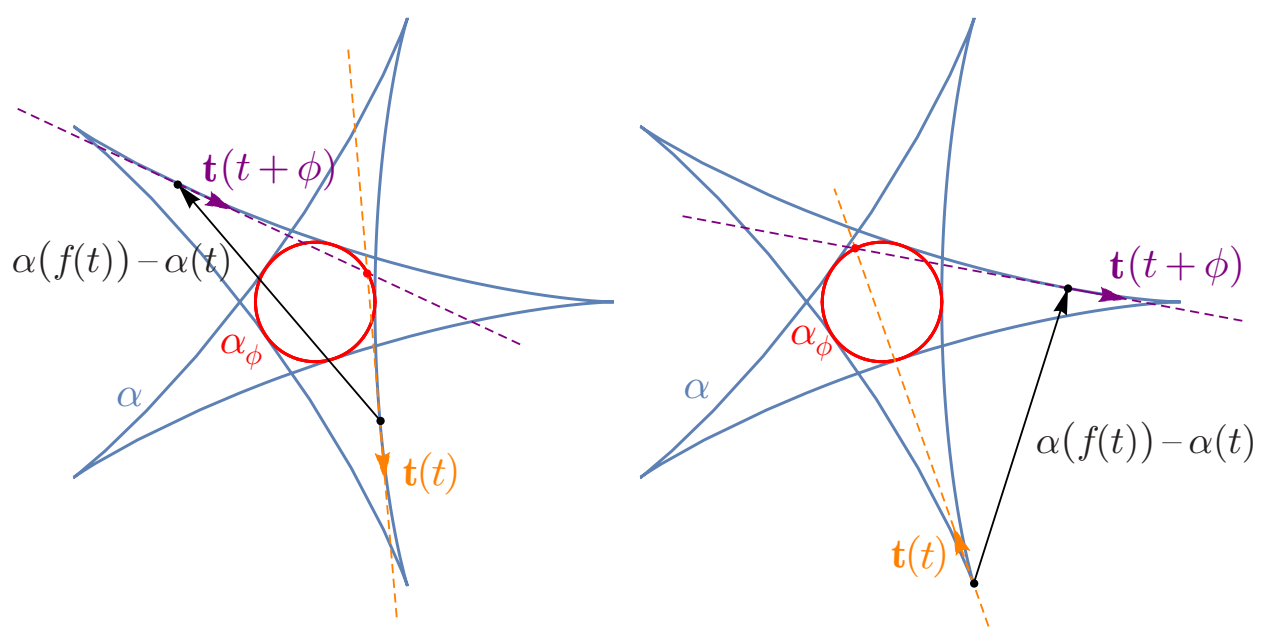

FiguRE 6 . The curve $\alpha$ is $(\pi / 3,3 \sqrt{3})$-isochordal viewed and it is different from a circle. When crossing each cusp, the angle between the tangent vectors varies between either $\phi$ or $\pi-\phi$.
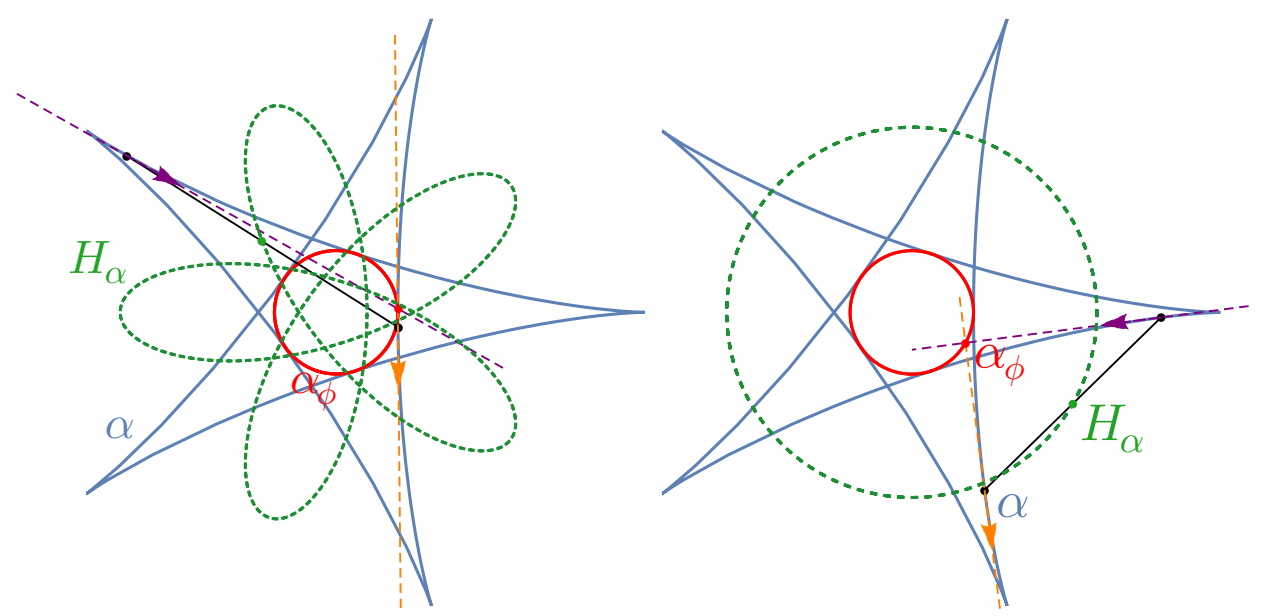

FiguRE 7. The curve $\alpha$, its $\phi$-isoptic $\alpha_{\phi}$ and its midpoint Holditch curve $H_{\alpha}$. On the left for $\phi=\pi / 3$ and on the right for $\phi=\pi / 2$.

but for $\phi=\pi / 2$, then the computations are quite easy and it can be shown that $\alpha$ is $(\pi / 2,4)$-isochordal viewed. In such a case, its $\phi$-isoptic is again a circle (clockwise and double traced), so that $\alpha$ is also a curve of constant $\pi / 2$-width. The midpoint Holditch curve is a clockwise and double-traced circle but of a greater radius (see Figure 7 rright). 
Example 3. Following the idea of the two examples above, we are going to give now a 1-parameter family of hedgehogs which are isochordal-viewed. Let $n \in \mathbb{N}, \phi \in] 0, \pi[$ and consider the family of hedgehogs

$$
\alpha_{n}(t)=h(t)(\cos t, \sin t)+h^{\prime}(t)(-\sin t, \cos t)
$$

defined by the support function $h(t)=\cos (n t)$.

It is straightforward to compute the squared distance function by

$$
\mathrm{d}_{2}(t)=\|\alpha(t+\phi)-\alpha(t)\|^{2} .
$$

Its derivative can be simplified and written as

$$
\mathrm{d}_{2}^{\prime}(t)=2 n\left(n^{2}-1\right)(\cos (n \phi)-\cos \phi) \sin (n(2 t+\phi)) .
$$

If we take $\phi=\pi / 2$, then

$$
\cos (n \phi)-\cos \phi=\cos \left(\frac{\pi n}{2}\right) .
$$

This equals zero if $n=2 k+1$ for any $k \in \mathbb{Z}$.

Therefore, the family of hedgehogs given by $\phi=\pi / 2$ and $n=2 k+1$, for all $k \in \mathbb{Z}$, are examples of isochordal-viewed curves. Moreover, they are also curves of constant $\phi$-width. Indeed, it can be seen (see Equation 14 below) that the curvature of the $\phi$-isoptic is constant. In particular (with the same notation as Equation 14 below), we have that

$$
A(t)=2-2(-1)^{k}(1+2 k) \text {. }
$$

A similar discussion can be done for other angles, for instance $\phi=\pi / 3$, where $n=5, n=7, n=11, n=13$, etc. also give examples of isochordalviewed curves.

\section{Integral FORMULAE AND AREAS OF ISOPTICS}

Given a curve $\alpha$, generate from $\alpha(t)$ another one at a distance $p(t)$ following directions given by an oriented angle function $w(t)$ with $\mathbf{t}(t)$ (see Figure 8).

Some properties of this generated curve from $\alpha$, say $\gamma$, have been given both in the plane (see [18]) and in constant curvature surfaces (see [19] and [15]) when the involved curves are closed and simple. Notice that if $\alpha$ is closed and $\gamma$ is wanted to be closed, then the functions $p$ and $w$ must be able to be extended continuously by periodicity.

Although it is common to assume simple closed curves to define areas, the notion of area can be extended to any non-simple closed curve as well. Given a closed curve $\alpha$ (not necessarily simple), the algebraic area of $\alpha$ is refereed as the area enclosed by $\alpha$ counted by sign and multiplicity. More specifically, the algebraic area of $\alpha$ is defined by

$$
\mathcal{A}(\alpha)=\iint_{\mathbb{R}^{2}} \operatorname{Wind}((x, y), \alpha) \mathrm{d}(x, y),
$$




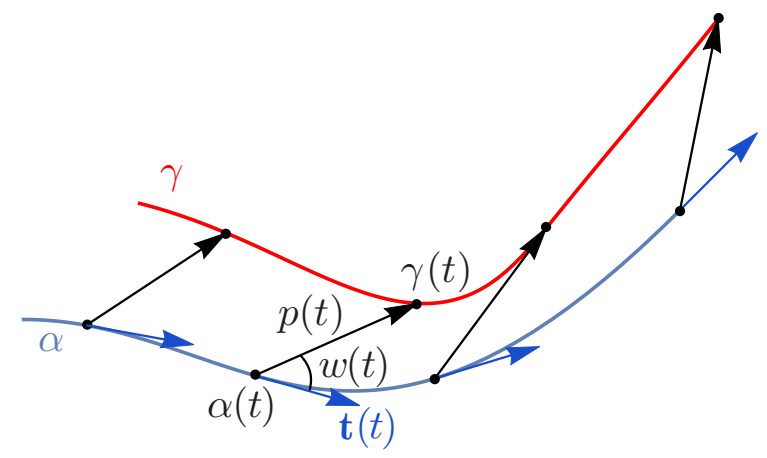

FigURE 8. Example of a curve $\gamma$ generated from $\alpha$ according to an angle function $w(t)$ and a distance function $p(t)$.

where $\operatorname{Wind}((x, y), \alpha)$ is the winding number of $\alpha$ around the point $(x, y) \in$ $\mathbb{R}^{2}$. Of course, if $\alpha$ is simple and counterclockwise oriented, we have

$$
\mathcal{A}(\alpha)=\iint_{D} \mathrm{~d}(x, y),
$$

where $D$ is the region of $\mathbb{R}^{2}$ enclosed by $\alpha$. Thus, in this case the algebraic area coincides with the geometric area (positive value).

In addition to Equation (7), thanks to the homological version of Green's theorem (see Theorem 6.8 of [2]), the algebraic area can also be computed with the usual expression:

$$
\mathcal{A}(\alpha)=\int_{\alpha} x \mathrm{~d} y
$$

Let's write now the general formula given in [18 for the area of a generated curve $\gamma$ in the plane but extended to any closed curve (not necessarily simple). We need this extension since, as seen in the examples above, the isochordal-viewed curves may not be simple. The proof is essentially the same but using the definition of an algebraic area.

Lemma 1. Let $\alpha: I \rightarrow \mathbb{R}^{2}$ be a piecewise-regular closed curve parameterized by arc-length. Suppose $\gamma$ to be the generated curve from $\alpha$ (where it can be defined) with a length function $p$ and directions given by a function $\beta: I \rightarrow$ $\mathbb{R}^{2}$ defined by

$$
\beta(s)=\cos w(s) \mathbf{t}(s)+\sin w(s) \mathbf{n}(s) .
$$

Suppose that $p$ and $\beta$ can be continuously extended by periodicity ( $\gamma$ is closed). Then

$$
\mathcal{A}(\gamma)=\mathcal{A}(\alpha)-\int_{I} p(s) \sin w(s) \mathrm{d} s+\frac{1}{2} \int_{I} p^{2}(s)\left(w^{\prime}(s)+\kappa(s)\right) \mathrm{d} s,
$$

where $w(s)$ is the oriented angle function from $\mathbf{t}(s)$ to $\beta(s)$ and $\kappa(s)$ the curvature function of $\alpha$. 
Notice that $\phi$-isoptics are a particular case of generated curves, where

$$
w(t)=0 \quad \text { and } \quad p(t)=\lambda_{1}(t, \phi)=\frac{d(t) \sin \mu(t)}{\sin \tilde{\phi}(t)} .
$$

Therefore, from Lemma 1, an integral formula relating the area of a $(\phi, \ell)$ isochordal-viewed curve $\alpha$ and the area of its $\phi$-isoptic $\alpha_{\phi}$ can be deduced.

Proposition 2. Let $\phi \in] 0, \pi[$ and let $\alpha$ be a piecewise-regular and closed $(\phi, \ell)$-isochordal-viewed curve parameterized by arc length. If $\alpha_{\phi}$ denotes the $\phi$-isoptic of $\alpha$, then

$$
\mathcal{A}\left(\alpha_{\phi}\right)=\mathcal{A}(\alpha)+\frac{\ell^{2}}{2 \sin ^{2} \phi} \int_{I} \sin ^{2} \mu(s) \kappa(s) \mathrm{d} s,
$$

where $\kappa$ is the curvature function of $\alpha$.

Proof. It is just an application of Lemma 1 for the functions

$$
w(s)=0 \quad \text { and } \quad p(s)=\lambda_{1}(s, \phi)=\frac{\ell \sin \mu(s)}{\sin \tilde{\phi}(s)}=\varepsilon(s) \frac{\ell \sin \mu(s)}{\sin \phi},
$$

where $\varepsilon$ is a sign function (see Remark 3). Of course, $\varepsilon^{2}(s)=1$.

In the next lemma, we show the relationship between $\kappa$ and $\kappa \circ f$.

Lemma 2. Let $\phi \in] 0, \pi\left[\right.$, let $\alpha: I \rightarrow \mathbb{R}^{2}$ be a piecewise-regular curve and let $f$ be the Holditch function for the parameterization $\alpha$ and the angle $\phi$. Then

$$
\kappa(t)\left\|\alpha^{\prime}(t)\right\|=\kappa(f(t))\left\|\alpha^{\prime}(f(t))\right\| f^{\prime}(t),
$$

for all $t \in I$ such that $\alpha$ is regular at $\alpha(t)$. In particular, if $\alpha$ is arc-length parameterized where it is regular, then

$$
\kappa(s)=\kappa(f(s)) f^{\prime}(s),
$$

for all $s \in I$ such that $\alpha$ is regular at $\alpha(s)$.

Proof. Let $\sigma: I \rightarrow \mathbb{R}$ be the oriented angle function from the positive $O X$ axis to the tangent $\mathbf{t}(t)$ of the curve $\alpha$ at $\alpha(t)$. By definition (see Section 2),

$$
\tilde{\phi}(t)=\sigma(f(t))-\sigma(t)
$$

for all $t \in I$ such that $\alpha$ is regular at $\alpha(t)$. The function $\tilde{\phi}(t)$ is piecewiseconstant, it is equal to $\phi$ or $\pi-\phi$ up to a multiple of $\pi$. Recall that $\sigma^{\prime}(t)=\left\|\alpha^{\prime}(t)\right\| \kappa(t)$. Thus, differentiating the expression (8), we get

$$
0=\kappa(f(t))\left\|\alpha^{\prime}(f(t))\right\| f^{\prime}(t)-\kappa(t)\left\|\alpha^{\prime}(t)\right\| .
$$

A similar integral formula to the one given in Proposition 2 can be derived but generating the $\phi$-isoptic of $\alpha$ from the parameterization $\alpha \circ f$ of the curve $\alpha$. 
Proposition 3. Let $\phi \in] 0, \pi[$ and let $\alpha$ be a piecewise-regular and closed $(\phi, \ell)$-isochordal-viewed curve parameterized by arc length. If $\alpha_{\phi}$ denotes the $\phi$-isoptic of $\alpha$, then

$$
\mathcal{A}\left(\alpha_{\phi}\right)=\mathcal{A}(\alpha)+\frac{\ell^{2}}{2 \sin ^{2} \phi} \int_{I} \sin ^{2} \nu(s) \kappa(s) \mathrm{d} s,
$$

where $\kappa$ is the curvature function of $\alpha$.

Proof. Let $f$ be the Holditch function for the parameterization $\alpha$ and the angle $\phi$. Let $s$ be the arc-length parameter of $\alpha$. Generate from $(\alpha \circ f)(s)$ the $\phi$-isoptic of $\alpha$ with the angle $w(s)=\pi$ and the distance function $p(s)=\lambda_{2}(s, \phi)$. Notice that $\kappa^{\alpha \circ f}(s)=\kappa(f(s))$ and that the new arc-length parameter of $\alpha \circ f$ is

$$
s^{\alpha \circ f}(s)=\int_{s_{0}}^{s} f^{\prime}(u) \mathrm{d} u .
$$

Hence, $\mathrm{d} s^{\alpha \circ f}=f^{\prime}(s) \mathrm{d} s$. Thus, from Lemma 1, we find

$$
\mathcal{A}\left(\alpha_{\phi}\right)=\mathcal{A}(\alpha)+\frac{\ell^{2}}{2 \sin ^{2} \phi} \int_{I} \sin ^{2} \nu(s) \kappa(f(s)) f^{\prime}(s) \mathrm{d} s .
$$

Finally, use Lemma 2 to write it as in the statement.

As a consequence of Propositions 2 and 3 we can find an integral formula involving the angle functions $\nu$ and $\mu$ and the curvature $\kappa$ of the curve.

Corollary 1. Let $\phi \in] 0, \pi[$. If $\alpha$ is a piecewise-regular and closed $(\phi, \ell)$ isochordal-viewed curve parameterized by arc length, then

$$
\int_{I}\left(\sin ^{2} \nu(s)-\sin ^{2} \mu(s)\right) \kappa(s) \mathrm{d} s=0 .
$$

Next, we will give the explicit value for the total sine of the angle function $\nu$ of a isochordal-viewed curve. Define the chord revolutions as the number of positive (counterclockwise) revolutions minus the number of negative (clockwise) revolutions done by the moving chord (seen as an indicatrix). If the chord comes back to its former position without making any full revolution, the chord revolutions are zero.

Theorem 1. Let $\alpha$ be a piecewise-regular and closed $(\phi, \ell)$-isochordal-viewed curve. Then

$$
\int_{I} \sin \nu(s) \mathrm{d} s=\pi \ell n
$$

where $n$ is the number of chord revolutions.

Proof. Suppose that $\alpha$ is arc-length parameterized in its regular parts. We will use Lemma 1 when the generated curve $\gamma$ is the parameterization $\alpha \circ f$, with $f$ being the Holditch function for the parameterization $\alpha$ and the angle 
$\phi$. Thus, with the notation of Lemma 1, we have $\mathcal{A}(\alpha)=\mathcal{A}(\alpha \circ f), p(s)=\ell$ and $w(s)=\nu(s)$. Therefore,

$$
\ell \int_{I} \sin \nu(s) \mathrm{d} s-\frac{\ell^{2}}{2} \int_{I}\left(\nu^{\prime}(s)+\kappa(s)\right) \mathrm{d} s=0 .
$$

Notice that

$$
\int_{I} \nu^{\prime}(s) \mathrm{d} s=2 \pi m, \quad \text { and } \quad \int_{I} \kappa(s) \mathrm{d} s=2 \pi I,
$$

where $m$ is the number of revolutions of the moving chord with respect to the tangent vector of $\alpha$ and $I$ is the rotation index of the regular parts of $\alpha$ (without taking into account the jump angles at the cusps). Thus, from Equation (10) we find

$$
\int_{I} \sin \nu(s) \mathrm{d} s=\pi \ell(m+I) .
$$

Finally, notice that $n=m+I$ is the number of chord revolutions.

Theorem 1 is a kind of Barbier-type theorem for isochordal-viewed curves. Note that the classical Barbier theorem for constant width curves can be deduced from Lemma 1 when $w(s)=\pi / 2$ and $p(s)=\ell$ in the same way as in the proof above. The fact is that for isochordal-viewed curves, the angle function $\nu$ may not be constant and, thus, the left-hand side of Equation (9) must be written in an integral form.

Example 4. Let's continue with the Example 1 to check the integral formulae given above. The curvature function of $\alpha$ is

$$
\kappa(t)=\frac{\left\langle\alpha^{\prime \prime}(t), J \alpha^{\prime}(t)\right\rangle}{\left\|\alpha^{\prime}(t)\right\|^{3}}=\frac{1}{8 \sqrt{\cos ^{2}(3 t)}} .
$$

In this example recall that $\phi=\pi / 2$ and $\ell=4$. Moreover, we have that

$$
\mathcal{A}(\alpha)=-2 \pi, \quad \text { and } \mathcal{A}\left(\alpha_{\phi}\right)=2 \pi .
$$

The formulae of Propositions 2 and 3 can be easily checked, since it can be seen explicitly that the integrals

$$
\mathcal{A}(\alpha)+\frac{\ell^{2}}{2 \sin ^{2} \phi} \int_{0}^{\pi} \sin ^{2} \mu(t) \kappa(t)\left\|\alpha^{\prime}(t)\right\| \mathrm{d} t
$$

and

$$
\mathcal{A}(\alpha)+\frac{\ell^{2}}{2 \sin ^{2} \phi} \int_{0}^{\pi} \sin ^{2} \nu(t) \kappa(t)\left\|\alpha^{\prime}(t)\right\| \mathrm{d} t
$$

are both equal to $2 \pi$, the area of the $\phi$-isoptic of $\alpha$.

The moving chord makes in $[0, \pi]$ a full clockwise revolution. This means that the chord revolutions here are $n=-1$. This number can be computed, as seen in the proof of Theorem 1 as the sum of the number of revolutions of the moving chord vector $\alpha(t+\pi)-\alpha(t)$ with respect to $\mathbf{t}(t)$ and the number 
$I$, which is the rotation index of the regular parts of $\alpha$. The first number can be computed as

$$
m=\frac{1}{2 \pi} \int_{0}^{\pi} \nu^{\prime}(t) \mathrm{d} t=-\frac{3}{2}
$$

and the second one as

$$
I=\frac{1}{2 \pi} \int_{0}^{\pi} \kappa(t)\left\|\alpha^{\prime}(t)\right\| \mathrm{d} t=\frac{1}{2} .
$$

Therefore, on the one hand, $n=m+I=-\frac{3}{2}+\frac{1}{2}=-1$ and the right-hand side of Equation (9) is $\pi \ell n=-4 \pi$. On the other hand, its left-hand side can be computed explicitly:

$$
\int_{0}^{\pi} \sin \nu(t)\left\|\alpha^{\prime}(t)\right\| \mathrm{d} t=-4 \pi
$$

so that Theorem 1 is checked in this example.

A similar discussion can be done for the curve of Example 2.

\section{Characterization for Curves of CONStant $\phi$-Width}

In the previous sections we have seen some examples of $(\phi, \ell)$-isochordalviewed curves constructed as hedgehogs by a support function $h$. It happened that all of them were examples of curves of constant $\phi$-width too. In this section we will prove a characterization on that fact in terms of the angle function $\nu$.

Let $\alpha:[0,2 \pi] \rightarrow \mathbb{R}^{2}$ be a regular hedgehog defined by a support function $h \in \mathcal{C}^{2}$ :

$$
\alpha(t)=h(t)(\cos t, \sin t)+h^{\prime}(t)(-\sin t, \cos t) .
$$

Recall that $h(t)$ is the signed distance from the origin to the supporting line of $\alpha$ with exterior normal vector $(\cos t, \sin t)$. The reader can see the works of Martínez-Maura on hedgehogs for a detailed discussion, e.g. [12].

In general, $\alpha$ is not a convex curve. We have that the radius $R$ of curvature verifies

$$
R(t)=\left\|\alpha^{\prime}(t)\right\|=\left|h(t)+h^{\prime \prime}(t)\right| .
$$

A singularity-free hedgehog is a convex curve. Thus, $\alpha$ is convex if and only if

$$
h(t)+h^{\prime \prime}(t)>0, \quad \text { for all } t \in[0,2 \pi] .
$$

To prove the characterization said above, we will need two lemmas.

Lemma 3. Let $\alpha: I \rightarrow \mathbb{R}^{2}$ be a curve satisfying the isochordal condition (2) for a Holditch function $f$ and a length $\ell>0$. Then

$$
\left\|\alpha^{\prime}(t)\right\| \cos \nu(t)=f^{\prime}(t)\left\|\alpha^{\prime}(f(t))\right\| \cos \mu(t) .
$$


Proof. The isochordal condition can be written as

$$
\|\alpha(f(t))-\alpha(t)\|^{2}=\ell^{2} .
$$

Differentiating this equation, we get

$$
f^{\prime}(t)\left\langle\alpha^{\prime}(f(t)), \alpha(f(t))-\alpha(t)\right\rangle=\left\langle\alpha^{\prime}(t), \alpha(f(t))-\alpha(t)\right\rangle .
$$

Thus,

$$
f^{\prime}(t)\left\|\alpha^{\prime}(f(t))\right\| \ell \cos \mu(t)=\left\|\alpha^{\prime}(t)\right\| \ell \cos \nu(t) .
$$

Since $\ell \neq 0$, we deduce the expression of the statement.

Lemma 4. Let $\phi \in] 0, \pi\left[\right.$ and let $\alpha: I \rightarrow \mathbb{R}^{2}$ be a piecewise-C $\mathcal{C}^{2}(\phi, \ell)$ isochordal-viewed hedgehog parameterized by a support function $h \in \mathcal{C}^{3}$. Then

$$
\ell\left(\nu^{\prime}(t)+1\right) \cos \mu(t)=\left\|\alpha^{\prime}(t)\right\| \sin \tilde{\phi}(t)
$$

for all $t \in I$ such that $\alpha$ is regular at $t$.

Proof. From the definition of $\nu$, we have:

$$
\langle\alpha(t+\phi)-\alpha(t), \mathbf{t}(t)\rangle=\ell \cos \nu(t) .
$$

Differentiating this expression and using

$$
\langle\alpha(t+\phi)-\alpha(t), \mathbf{n}(t)\rangle=\ell \sin \nu(t),
$$

we get

$$
\left\|\alpha^{\prime}(t+\phi)\right\| \cos \tilde{\phi}(t)-\left\|\alpha^{\prime}(t)\right\|+\kappa(t)\left\|\alpha^{\prime}(t)\right\| \ell \sin \nu(t)=-\ell \sin \nu(t) \nu^{\prime}(t) .
$$

This can be rewritten as

$$
\ell \sin \nu(t)\left(\nu^{\prime}(t)+1\right)=\left\|\alpha^{\prime}(t)\right\|-\left\|\alpha^{\prime}(t+\phi)\right\| \cos \tilde{\phi}(t),
$$

where it has been used the fact:

$$
\kappa(t)\left\|\alpha^{\prime}(t)\right\|=1
$$

Now, following the same procedure but for the equation

$$
\langle\alpha(t+\phi)-\alpha(t), \mathbf{t}(t+\phi)\rangle=\ell \cos \mu(t),
$$

we arrive to

$$
\ell \sin \mu(t)\left(\mu^{\prime}(t)-1\right)=\left\|\alpha^{\prime}(t)\right\| \cos \tilde{\phi}(t)-\left\|\alpha^{\prime}(t+\phi)\right\| .
$$

Subtracting to 11) Equation 12 multiplied by $\cos \tilde{\phi}(t)$, we get

$$
\ell \sin \nu(t)\left(\nu^{\prime}(t)+1\right)-\ell \sin \mu(t) \cos \tilde{\phi}(t)\left(\mu^{\prime}(t)-1\right)=\left\|\alpha^{\prime}(t)\right\| \sin ^{2} \tilde{\phi}(t) .
$$

Now, since $\tilde{\phi}$ is piecewise-constant, $0=\tilde{\phi}^{\prime}(t)=\nu^{\prime}(t)+\mu^{\prime}(t)$, so that $\mu^{\prime}(t)=$ $-\nu^{\prime}(t)$. Using this in the equation above, we get

$$
\ell\left(\nu^{\prime}(t)+1\right)(\sin \nu(t)+\sin \mu(t) \cos \tilde{\phi}(t))=\left\|\alpha^{\prime}(t)\right\| \sin ^{2} \tilde{\phi}(t) .
$$

Equivalently,

$$
\ell\left(\nu^{\prime}(t)+1\right) \cos \mu(t) \sin \tilde{\phi}(t)=\left\|\alpha^{\prime}(t)\right\| \sin ^{2} \tilde{\phi}(t) .
$$


Finally, since $\phi \in] 0, \pi[$, by definition of $\tilde{\phi}$ we have that $\sin \tilde{\phi}(t) \neq 0$ for all $t \in I$. Thus, by dividing 13 by $\sin \tilde{\phi}(t)$, the expression of the statement is found.

Proposition 4. Let $\alpha$ be a piecewise-C $\mathcal{C}^{2}(\phi, \ell)$-isochordal-viewed hedgehog parameterized by a support function $h \in \mathcal{C}^{3}$. Then the curvature of the $\phi$-isoptic of $\alpha$ is

$$
\kappa_{\alpha, \phi}(t)=\frac{\sin \tilde{\phi}(t)}{\ell}\left(1-\nu^{\prime}(t)\right)
$$

for all $t \in I$ such that $\alpha$ is regular at $t$.

Proof. Since $\tilde{\phi}^{\prime}(t)=0$, the expression (6) for the curvature of the $\phi$-isoptic of a curve $\alpha$ still works for non-convex curves. This curvature can be written as

$$
\kappa_{\alpha, \phi}(t)=\frac{\sin \tilde{\phi}(t)}{\ell^{3}}\left(2 \ell^{2}-A(t)\right)
$$

where

$$
\begin{aligned}
A(t) & =\left\langle\alpha(t+\phi)-\alpha(t), J \alpha^{\prime}(t)-J \alpha^{\prime}(t+\phi)\right\rangle \\
& =\ell\left\|\alpha^{\prime}(t)\right\| \sin \nu(t)+\ell\left\|\alpha^{\prime}(t+\phi)\right\| \sin \mu(t) .
\end{aligned}
$$

By Lemma 3 .

$$
\left\|\alpha^{\prime}(t)\right\| \cos \nu(t)=\left\|\alpha^{\prime}(t+\phi)\right\| \cos \mu(t)
$$

This implies that if $\cos \mu\left(t_{0}\right)=0$ for some $t_{0} \in I$, then $\left\|\alpha^{\prime}\left(t_{0}\right)\right\|=0$ (because $\cos \nu\left(t_{0}\right) \neq 0$, otherwise $\phi=0$ or $\left.\pi\right)$. Therefore, if we are at a regular point $t_{0}$ of $\alpha$ (not at a cusp), then $\cos \mu\left(t_{0}\right) \neq 0$.

Thus, if $t \in I$ is such that $\alpha$ is regular at $t$, from Equation (15):

$$
\left\|\alpha^{\prime}(t+\phi)\right\|=\left\|\alpha^{\prime}(t)\right\| \frac{\cos \nu(t)}{\cos \mu(t)} .
$$

Substituting this in the expression of $A(t)$ an simplifying, it is deduced

$$
A(t)=\ell\left\|\alpha^{\prime}(t)\right\| \frac{\sin \tilde{\phi}(t)}{\cos \mu(t)}
$$

Now, use Lemma 4 to get

$$
A(t)=\ell^{2}\left(1+\nu^{\prime}(t)\right) .
$$

Therefore, Equation (14) takes the form

$$
\begin{aligned}
\kappa_{\alpha, \phi}(t) & =\frac{\sin \tilde{\phi}(t)}{\ell^{3}}\left(2 \ell^{2}-\ell^{2}-\ell^{2} \nu^{\prime}(t)\right) \\
& =\frac{\sin \tilde{\phi}(t)}{\ell}\left(1-\nu^{\prime}(t)\right)
\end{aligned}
$$

as in the statement. 
Immediately from Proposition 4, we have the following result, which shows when an isochordal-viewed hedgehog is a curve of constant $\phi$-width.

Theorem 2. Let $\alpha$ be a piecewise- $\mathcal{C}^{2}(\phi, \ell)$-isochordal-viewed hedgehog parameterized by a support function $h \in \mathcal{C}^{3}$. The curve $\alpha$ is of constant $\phi$-width if and only if $\nu^{\prime}(t)$ is constant for all $t \in I$.

For the curves of Examples 1 and 2, the angle function $\nu$ is piecewiselinear, namely, of the kind $\nu(t)=a t+b$, with the same slope where it can be defined. This implies that $\nu^{\prime}(t)=a$ is constant, so that by Theorem 2, they are curves of constant $\phi$-width.

Note that since $\nu^{\prime}(t)=-\mu^{\prime}(t)$, the conclusion of Theorem 2 could be also written in terms of the angle function $\mu(t)$.

Remark 4 (Open problems). The author has not found any explicit example of a $(\phi, \ell)$-isochordal-viewed hedgehog which is not a curve of constant $\phi$-width. In fact, no example of isochordal-viewed curve which is not an hedhehog has been found. It would be interesting to find such examples if they exist or to see if a statement like "any piecewise- $\mathcal{C}^{2}(\phi, \ell)$-isochordal-viewed curve (or hedgehog) is a curve of constant $\phi$-width" is true.

\section{REFERENCES}

1. Arne Broman, Holditch's theorem, Math. Mag. 54 (1981), no. 3, 99-108.

2. Joaquim Bruna and Julià Cufí, Complex analysis, EMS Textbooks in Mathematics, European Mathematical Society (EMS), Zürich, 2013, Translated from the Catalan by Ignacio Monreal.

3. Waldemar Cieślak, Horst Martini, and Witold Mozgawa, On Holditch's theorem, J. Geom. 111 (2020), no. 2, 24.

4. Waldemar Cieślak, Andrzej Miernowski, and Witold Mozgawa, Isoptics of a closed strictly convex curve, Global differential geometry and global analysis (Berlin, 1990), Lecture Notes in Math., vol. 1481, Springer, Berlin, 1991, pp. 28-35.

5. Isoptics of a closed strictly convex curve. II, Rend. Sem. Mat. Univ. Padova 96 (1996), 37-49.

6. Mark J. Cooker, An Extension of Holditch's Theorem on the Area within a Closed Curve, The Mathematical Gazette 82 (1998), no. 494, 183-188.

7. Thierry Dana-Picard, An automated study of isoptic curves of an astroid, J. Symbolic Comput. 97 (2020), 56-68.

8. Thierry Dana-Picard and Witold Mozgawa, Automated exploration of inner isoptics of an ellipse, J. Geom. 111 (2020), no. 2, Paper No. 34, 10.

9. John W. Green, Sets subtending a constant angle on a circle, Duke Math. J. 17 (1950), 263-267.

10. Hamnet Holditch, Geometrical theorem, The Quarterly J. Pure Appl. Math 2 (1858), 38.

11. Jesús Jerónimo-Castro, Francisco G. Jimenez-Lopez, and A. Rosario Jiménez-Sánchez, On convex bodies with isoptic chords of constant length, Aequationes Math. 94 (2020), no. 6, 1189-1199.

12. Yves Martinez-Maure, Geometric inequalities for plane hedgehogs, Demonstratio Math. 32 (1999), no. 1, 177-183. 
13. Shigetake Matsuura, On nonconvex curves of constant angle, Functional analysis and related topics, 1991 (Kyoto), Lecture Notes in Math., vol. 1540, Springer, Berlin, 1993, pp. 251-268.

14. Juan Monterde and David Rochera, Holditch's ellipse unveiled, Amer. Math. Monthly 124 (2017), no. 5, 403-421.

15. (2020), no. 4, 20 pp.

16. Witold Mozgawa, Mellish theorem for generalized constant width curves, Aequationes Math. 89 (2015), no. 4, 1095-1105.

17. Harald Proppe, Alina Stancu, and Ronald J. Stern, On Holditch's theorem and Holditch curves, J. Convex Anal. 24 (2017), no. 1, 239-259.

18. Luis A. Santaló, Area bounded by the curve generated by the end of a segment whose other end traces a fixed curve, and application to the derivation of some theorems on ovals, Math. Notae 4 (1944), 213-226 (in Spanish).

19. Enrique Vidal Abascal, Area generated on a surface by an arc of a geodesic when one of its ends describes a fixed curve and length of the curve described by the other end, Revista Mat. Hisp.-Amer. (4) 7 (1947), 132-142 (in Spanish).

E-mail address: drochera@bcamath.org

Alameda de Mazarredo, 14, E-48009 Bilbao (Basque Country), Spain 